\title{
Multi-parametric Flow Cytometry for Neuroblastoma, a new and possible diagnostic tool. Case report
}

\author{
Belén Manrique, Biochemist ${ }^{a}$ Jessica López Marti, M.D. ${ }^{c}$, Walter Cacciavillano, M.D. ${ }^{b}$ and Jorge Rossi, M.D. ${ }^{a}$
}

\begin{abstract}
Neuroblastoma is the most frequent extracranial solid tumor in childhood, representing $5.6 \%$ according to the "Registro Oncopediátrico Hospitalario Argentino". For its diagnosis, several complementary methods (radiological, biological and biochemical) are required, and Multi-parametric Flow Cytometry (MFC) arises as a potential diagnostic method, despite not having been so far extensively explored. MFC is a method that allows to obtain several information about size, internal complexity and antigenic expression by the use of a laser and fluorescent monoclonal antibodies. There are an increasing number of reports in the literature, which reveal the importance of using MFC for diagnosis and monitoring of solid tumors. The aim in this presentation is to highlight the fundamental role that MFC had in the case of a patient affected by neuroblastoma, in which an early diagnosis using this methodology allowed prompt administration of adequate treatment.

Key words: neuroblastoma, flow cytometry, non-Hematopoietic neoplasms, children, and neoplasms.
\end{abstract}

http:/ /dx.doi.org/10.5546/aap.2016.eng.e100

\section{INTRODUCTION}

Neuroblastoma is the most frequent extra cranial solid tumor in childhood, representing $5.6 \%$ of all pediatric malignant tumors in Argentina according to the "Registro Oncopediátrico Hospitalario Argentino" (ROHA: results 2000-2008).

The origin of this tumor is neural cells crest and it is usually located in abdomen and kidney glands. It can also be found in neck, thorax and pelvis. The most common clinical presentation is by the presence of a tumor mass.

The prognostic is variable and depends on several factors, such as age of presentation, histological subtype, and the presence or absence of biological factors (amplification of the MYCN

a. Immunology and Rheumatology,

b. Hematology and Oncology

c. Pathology Departments.

Hospital de Pediatría "Prof. Dr. Juan P. Garrahan".

E-mail Address:

Belén Manrique: belen.manrique@gmail.com

Funding: none.

Conflict of interest: none.

Received: 7-25-2015

Accepted: 10-5-2015 oncogene, 17 q gaining, deletion 1p36 and deletion $11 \mathrm{q})$. The disseminated NB diagnosed after the year of age is always highly aggressive, with a poor prognostic.

For its diagnosis, several complementary methods (radiological, biological and biochemical) are required, and multi-parametric flow cytometry arises as a potential diagnostic method, despite not having been so far extensively explored. MFC is a method that allows to obtain several information about size, internal complexity and antigenic expression by the use of a laser and fluorescent monoclonal antibodies. MFC has been used mostly for diagnosis and monitoring of hematologic malignancies, but not in non-hematopoietic tumors (solid tumors). In the latter, its use has been limited mostly to the determination of DNA index with prognosis purposes, but its potential application with diagnostic purposes is yet to be determined. It will possibly allow identification of tumor cells in a quick way, even in samples with scarce cellularity. Furthermore, the availability of new cell markers will expand the role of MFC as a diagnostic tool. ${ }^{1}$

In a recent article, different types of pediatric solid tumors were evaluated by MFC with a panel of markers for diagnostic screening and subsequent sub classification. The purpose of the authors was to distinguish between tumors infiltrated samples and reactive samples, hematologic malignancies versus non hematologic neoplasias, and even to establish differential diagnoses between the different solid tumors. ${ }^{2}$

Outcome for NB patients is variable and is related to several factors such as age, stage of the tumor, histology and cytogenetic/molecular changes, among which are the amplification of the MYCN oncogene, $17 \mathrm{q}$ gaining, deletion $1 \mathrm{p} 36$ and deletion $11 \mathrm{q}$. Disseminated NB diagnosed in patients older than one year of age are always highly aggressive, with unfavorable prognosis.

MFC has the advantage of providing immediate results, with subsequent correlation with the histologycal/pathology diagnosis, a fact relevant in cases in which the clinical status of patients requires effective treatment onset immediately. 
In this article we report a clinical case in which the MFC findings had diagnostic relevance.

\section{CLINICAL PRESENTATION}

A nine months old female patient, transferred from another institution, was admitted at Garrahan Hospital on 09-29-2013. She presented a cervical tumor of three weeks of evolution with rapid growth on the days prior to consultation. The initial presumptive diagnosis was acute leukemia based in the report of a bone marrow aspiration (BMA) morphology evaluation from the institution which transferred the patient which acknowledged $62 \%$ of myeloid blast infiltration. On admittance, signs of respiratory difficulty due to the tumor's mechanical compression were verified, therefore endotracheal intubation was set. A new BMA was performed in order to confirm the reported results before starting treatment.
The bone marrow sample was analyzed by MFC, using a panel of antibodies with the purpose of confirming acute leukemia. Surprisingly, an amount of nearly $47 \%$ of cells with neither expression of CD45 nor of any of the hematopoietic lineage specific antigens but positivity for CD56 was detected. These results ruled out acute leukemia and oriented the diagnosis to a non-hematopoietic tumor which has this pattern of antigenic expression. Thus, further markers related to solid tumors were evaluated, such as CD90, which resulted positive (Figure 1). Disialoganglioside GD2 was also evaluated by optical microscopy using indirect inmunofluorescence (IIF) disclosing an also positive result (Figure 2). These findings led oncologists to rule out acute leukemia and to consider NB as the most probable diagnosis.

Taking into account these findings and due to the severe clinical condition of the patient,
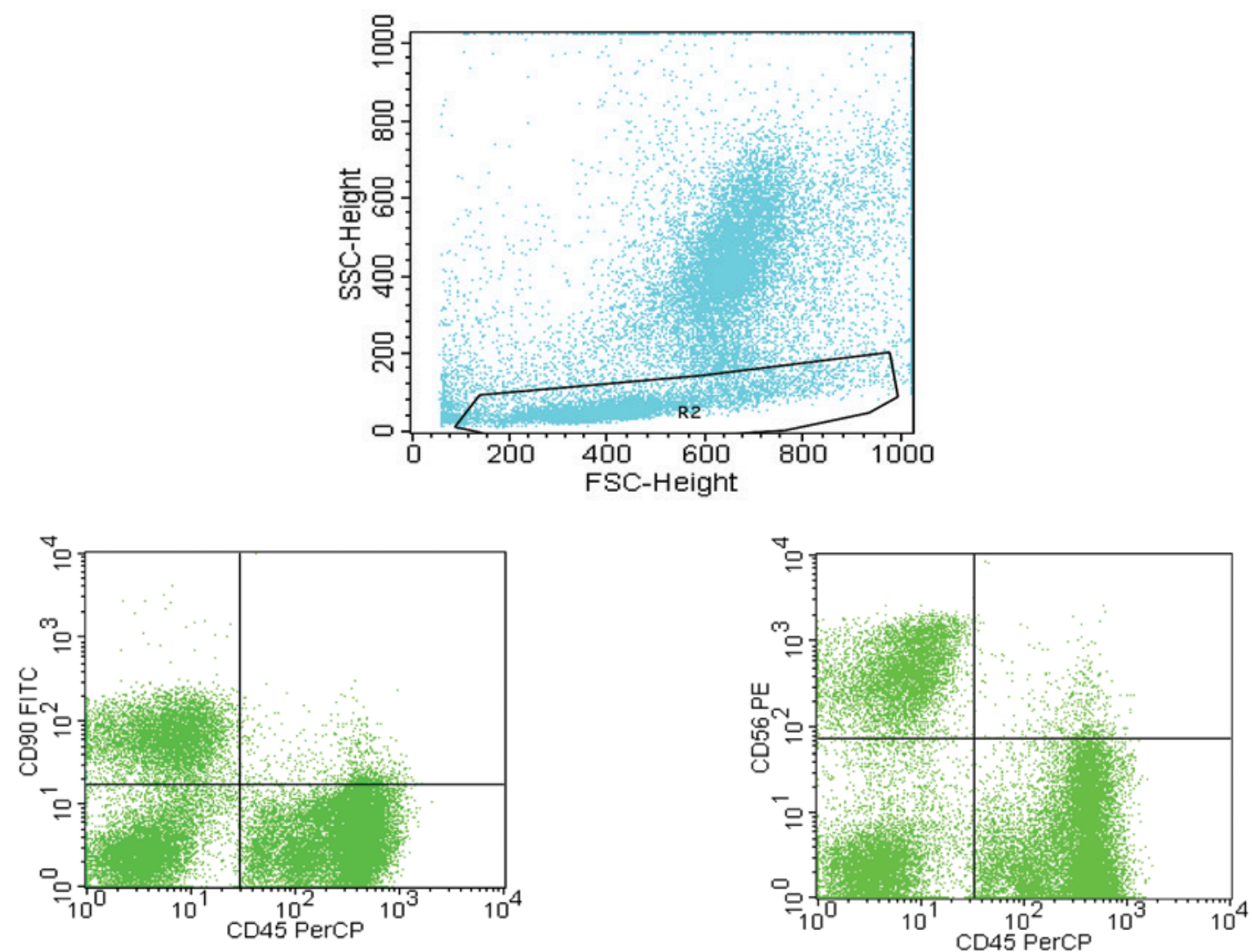

Bone marrow sample analyzed by MFC, dated 09/29/2013. A population around $47 \%$ is observed in the lympho-mononuclear region in size versus complexity graphic (R2). Analyzing the cells gated in R2, in the CD56 (PE) versus CD45 (PerCp) graphic, we observed that they do not express CD45 but they do express CD56. Analyzing R2 population in the CD90/FITC) versus CD45 (PerCp) graphic, we observed that they express CD90. The population selected in $\mathrm{R} 2$ have the following phenotype of expression: CD45-/CD56+/CD90+ and represents a $47 \%$ of the total population in the BM. 
chemotherapy with carboplatin $6.6 \mathrm{mg} / \mathrm{kg} /$ day and etoposide $5 \mathrm{mg} / \mathrm{kg} /$ day during three days was started for initial treatment.

Then, staging of the patient was evaluated considering CT scanning and scintilliography with metaiodobenzylguanidine.

On $10 / 16 / 2013$, a biopsy of the tumor was performed and the pathology evaluation confirmed the NB diagnosis, poorly differentiated, with an index of mitosis karyorrhexis above $4 \%$, thus representing an unfavorable histology. Amplification of oncogene N-myc and deletion 1p36It was disclosed by interface FISH.

The patient was considered as high risk because she was younger than 18 months of age, stage $M$ according to the International Neuroblastoma Risk Group Staging System (INRGSS), ${ }^{3}$ unfavorable histology, amplification of oncogene N-myc and deletion $1 \mathrm{p} 36$.

After the initial cycle, the patient continued the treatment with induction combination chemotherapy being refractory, and dying as result of the disease progression after six months of diagnosis.

\section{DISCUSSION}

NB is the extra cranial solid tumor more frequent in childhood. This malignancy is

FIGURE 2. Indirect staining of GD2 by fluorescence microscopy

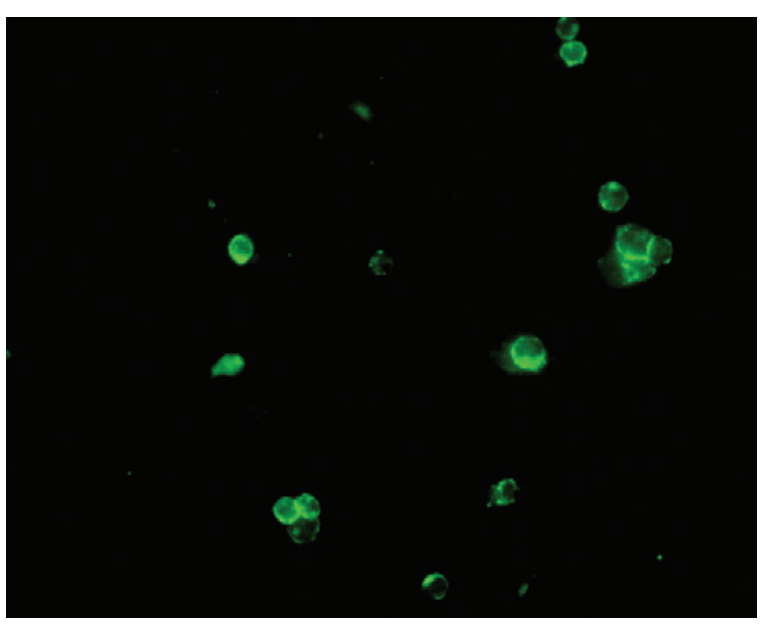

IFI of GD2 by optic microscopy on BM mononuclear cells. We observed cells, isolated and in groups, characteristic of NB showing an intense and continued positivity along the plasmatic cell membrane. This technique was realized by an indirect labeling using as primary antibody an antiganglioside (3F8) and as secondary antibody an anti-mouse labeled with FITC. originated at the cells of the neural crest. It is located more frequently at abdominal level (adrenal glands), but it can have other locations as neck, thorax and pelvis. The prognostic is variable and depends on several factors, such as age of presentation, histological subtype, and the presence or absence of biological factors (amplification of the MYCN oncogene, $17 \mathrm{q}$ gaining, deletion $1 \mathrm{p} 36$ and deletion $11 \mathrm{q}$ ).

The clinical course of NB is also variable; the tumor can experience spontaneous regressions, differentiation into mature components, responding, progressing or being refractory to the conventional treatments.

NB is classified in low, intermediate and high risk according to: age of presentation, location, radiological findings, biological factors and pathology $y^{3}$. The patients classified as low and intermediate risk have a very good prognosis when they are treated with chemotherapy, surgery and radiotherapy. In high risk patients (over 18 months old) who experienced a metastatic disease or with amplification of the MYCN oncogene, the treatment approaching is multimodal: chemotherapy in conventional or higher doses, surgery, radiotherapy, maintenance with retinoic acid and/or immunotherapy. Despite treatment intensification, the prognosis continues to be poor, with survivals lesser than $40 \%{ }^{4}$. Children with this type of presentation are the main group among patients with NB diagnoses. While half of the cases present with localized disease and have high curative chances, another half experience disseminated disease and have an unfavorable survival prognosis (except for patients younger than 18 months old).

Early diagnosis in oncology is very important because it determines the initial therapeutic decision.

Lately, the knowledge about biomarkers in cancer has increased dramatically, generating new tools for improving patients' handling by increasing efficiency of detection and therefore the efficacy of treatment. These biomarkers include a broad range of biochemical entities such as nucleic acids, proteins, carbohydrates, lipids and metabolites, cytogenetic and cytokinetic parameters, as well as cells detected in body fluids. A keen understanding of the relevance in each biomarker is very important, not only for a reliable diagnosis, but also for collaborating in choosing the best available treatments.

Use of MFC in malignancies has been expanded significantly during recent years. For 
a long time it has been used for the definition of antigenic profiles in leukemia and lymphoma, establishing thus the lineage and maturation stage of blasts, information critical for treatment decision making. Later, determination of minimal residual disease (MRD) was incorporated to treatment protocols, setting more precise risk group definitions and finally resulting in better survival rates. ${ }^{5}$

Actually there is evidence of the utility of MFC in diagnostic and evaluation of minimal disseminated disease in BM, as for the follow up and evaluation of minimal residual disease, through the optimal marker combination. ${ }^{6,7}$

It is know that the antigen expression profile of NB is: CD45-/CD81+/CD9+/CD90+/GD2+. ${ }^{2}$

In this case the detection of solid tumor cells by MFC started by the finding of a CD45negative population, which is pan-leukocyte marker, present in cells of hematopoietic origin. Therefore, non-hematopoietic tumor cells do not express this antigen.

Among the markers evaluated in solid tumor definition, CD56 is the most studied. Despite most of these tumors express it, intensity varies between the different tumor types. NB and PNET (Primitive Neuroectodermal Tumors) show the highest levels of CD56.

GD2 is a disialoganglioside expressed in cells of neuroectodermal origin; therefore its reflection is linked to NB and PNET. The expression of GD2 in NB is distinctively intense.

CD 90 is a stem cell like marker and also expressed in proliferating cells, and being expressed in NB cells.

\section{CONCLUSION}

In summary, in the field of solid tumors, MFC will constitute a methodology that will enable achieving results promptly, thus leading to a diagnosis and onset of the right treatment in an early stage. Furthermore, the evaluation of tumor infiltrating cells in BMA at diagnosis will help to define the initial staging; and during the treatment it will define together with other methods the response to it, through the evaluation of minimal residual disease or early detection of relapses. Further studies in a prospective cohort of patients with pediatric solid tumors are necessary to definitely establish the contribution of this methodology to these neoplasms in order to include MFC as one of the diagnostic and monitoring tools in solid tumors.

\section{REFERENCES}

1. Varma N, Naseem S. Application of flow cytometry in pediatric hematology-oncology. Pediatr Blood Cancer 2011;57(1):18-29.

2. Ferreira-Facio CS, Milito C, Botafogo V, Fontana M, et al. Contribution of multiparameter flow cytometry immunophenotyping to the diagnostic screening and classification of pediatric cancer. PLoS One 2013;8(3):e55534.

3. Cohn SL, Pearson AD, London WB, Monclair T, et al. The International Neuroblastoma Risk Group (INRG) classification system: an INRG Task Force report. J Clin Oncol 2009;27(2):289-97.

4. Matthay KK, Reynolds CP, Seeger RC, Shimada H, et al. Long term results for children with high risk neuroblastoma treated on a randomized trial of myeloablative therapy followed by 13-cis-retinoic acid: a children's oncology group study. J Cin Oncol 2009;27(7):1007-13.

5. Basso G, Veltroni M, Valsecchi MG, Dworzak MN, et al. Risk of relapse of childhood acute lymphoblastic leukemia is predicted by flow cytometric measurement of residual disease on day 15 bone marrow. J Clin Oncol 2009;27(31):5168-74.

6. Bozzi F, Gambirasio F, Luksch R, Collini P, et al. Detecting CD56+/NB84+/CD45- inmunophenotype in the bone marrow of patients with metastatic neuroblastoma using flow cytometry. Anticancer Res 2006;26(5A):3281-87.

7. Köksal Y, Reisli I, DoguF, Ucar C, et al. Can flow-cytometry be used at diagnosis and follow-up in neuroblastoma with bone marrow involvement? Turkish Journal of Cancer 2006:36(1):27-30. 